\title{
Numerical modeling of train aerodynamics in a straight tunnel
}

\author{
Sergio Croquer \\ Mechanical Engineering Department \\ Université de Sherbrooke \\ Sherbrooke, Canada \\ Sergio.Croquer.Perez@USherbrooke.ca
}

\author{
Hachimi Fellouah \\ Mechanical Engineering Department \\ Université de Sherbrooke \\ Sherbrooke, Canada \\ Hachimi.Fellouah@USherbrooke.ca
}

\author{
Sébastien Poncet \\ Mechanical Engineering Department \\ Université de Sherbrooke \\ Sherbrooke, Canada \\ Sebastien.Poncet@USherbrooke.ca
}

\begin{abstract}
A numerical investigation using $k-\omega$ SST RANS modeling has been carried out, to evaluate the aerodynamics of different metro train geometries through a straight tunnel. Drag performance has been compared with a typical blunt face train design, representative of European metro networks. The proposed models have different edge rounding characteristics at the front and rear faces. The study focuses on skin friction and pressure drag coefficients for trains traveling at $40 \mathrm{~km} / \mathrm{hour}$ in a straight tunnel with a blockage ratio of 0.69 . All the considered alternatives show a drag reduction of at least $5 \%$ relative to the baseline case.
\end{abstract}

Index Terms-metro train performance, drag reduction, RANS modeling

\section{INTRODUCTION}

Since the inauguration of the first railway in London in 1863, underground transportation has been a key element for the development of dense urban areas [1]. Being more punctual, cheaper and safer than their surface counterparts, metro networks rapidly become the preferred method of transportation for growing cities [2]. They are also an effective way for a city to meet its pollution targets. For instance, it is estimated that a $3 \%$ increase in the commuter share of the public network of Montréal (Québec, Canada) would reduce the city yearly $\mathrm{CO}_{2}$ emissions by 54000 tons.

Thus, a great interest has risen in the last few decades for developing better underground networks, able to make densely populated areas more habitable while at the same time reducing operational costs. Given the typical system dimensions and flow complexity, an important share of these efforts focuses on employing numerical methods rather than experimental techniques. One dimensional models of unsteady flows in train tunnels have been developed since the early eighties [3], mainly for characterization of pressure and temperature variations with passing trains. In the last decades, the Reynolds-Averaged Navier-Stokes (RANS) approach with the $k-\epsilon$ turbulence model has been applied to study more detailed phenomena such as the piston effect [2], i.e., the important currents generated in the tunnels as the air is pushed by the front of the train and sucked by the rear. It has been determined that overall energy savings of $3 \%$

Support from Clean Rail Academic Grant Program (Transport Canada, Government of Canada) and HPC facilities of Compute Canada might be achieved if the piston-effect is properly harvested for platform ventilation [4], although at higher train speeds traininduced pressure fluctuations might compromise the station structure [5]. More complex approaches such as Detached Eddy Simulation (DES) and Large-Eddy Simulation (LES) have been successfully used in aerodynamic studies of smaller vehicles [6]. As well as in specific aspects of trains, e.g., the flow structures around train boogies and the conditions which promote ballast flight [7] and in the assessment of pollutant dispersion with train-induced airflow in a two-story subway platform [8]. However, their computational costs are still impractical for the aerodynamic analysis of a complete metro train.

In order to reduce the energy footprint of metro networks, attention should be addressed now towards specific issues such as the aerodynamic performance of the metro trains. Typically, studies on train aerodynamics are more concerned with high speed railways, where elevated velocities imply considerable drag losses [9] and complex flow features both in open air conditions [10] and when entering tunnels [11]. RANS techniques combined with shape optimization algorithms have been used to determine the optimal streamlined geometry for high speed trains [12]. Yet, aerodynamic performance of metro networks has not gained too much attention in the past given the low speed train velocities, even though motion resistance represents $16 \%$ of the traction energy invested in a typical system [4]. Moreover, the typical metro blunt shape and high blockage ratio tend to emphasize pressure fluctuations on metro platforms. Though these important issues need to be addressed, there are very few studies on improving the aerodynamic performance of subway trains.

Thus, with the objective of providing alternatives to enhance the performance of underground trains, this work presents for the first time a numerical study of various in-house train designs conceived specifically for metro networks. A train geometry typical of rolling stocks used in various European cities has been defined as baseline against which 7 design variations are compared in terms of the pressure and friction coefficients and the overall aerodynamic performance. The assessed alternatives cover in particular the curvature of the train front and rear faces. Further details and dimensions of the assessed cases are provided in the following, with a description 
of the numerical model given next. Results are then presented and discussed, followed by the most relevant conclusions.

\section{TRAIN DESIGNS}

A blunt train geometry based on typical underground railway rolling stocks currently used in various European cities was chosen as baseline (Case $A$ ). The original CAD file was taken from a public on-line repository and it is based on the U5 fleet of the Vienna (Austria) subway system. The tunnel was constructed around this geometry, respecting typical single lane width and height values. The train consists of three motorized/passenger cars with straight angled front and rear faces. Its dimensions expressed as height, twice the half-width and length, are respectively: $H_{t}=4.5 \mathrm{~m}, 2 W_{t}=3.5 \mathrm{~m}$ and $L_{t}=53.6 \mathrm{~m}$. Each cart has a length $L_{c}=18.3 \mathrm{~m}$. A general view of the baseline train design is shown in Fig. 1.
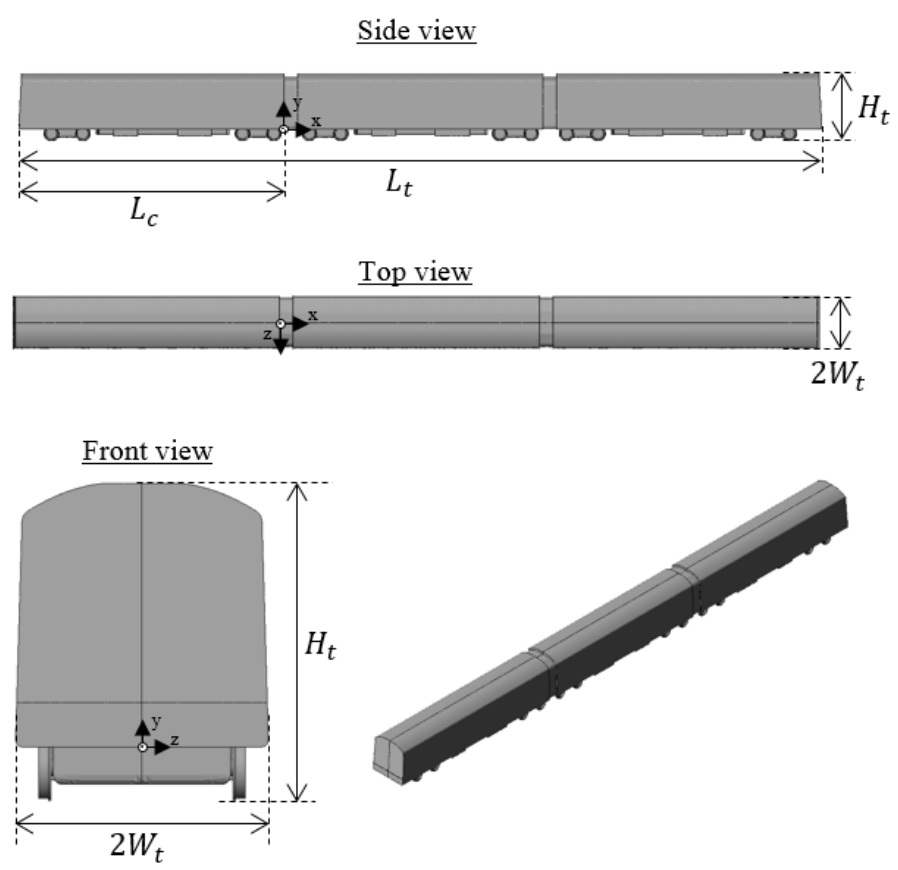

Fig. 1. Dimensions of the baseline train geometry (Case A).

A series of modifications to the baseline design (Case $A$ ) have been considered, the objective being to reduce the total drag without a noticeable sacrifice in internal space and keeping the same train dimensions (i.e. $H_{t} \times W_{t} \times L_{t}$ ). Cases $B$, $C$ and $D$ present modifications to the baseline wherein the top and bottom edges of the train are rounded at different degrees. These profiles are depicted in Fig. 2 with the construction points indicated in Tab. I. Moreover, cases $A \_s i d e s, B \_s i d e s$ and $C_{-}$sides repeat their respective profiles, but with rounded side edges. This modification was not applied to case $D$ as it would imply an important reduction in internal usable space. Lastly, case $A \_s k i r t$ is the same geometry as case $A$, but with an added panel underneath, similar to the trailer skirts used to reduce aerodynamic drag in trucks [13]. The schematics and construction points of the straight sides, rounded sides and skirt profiles are given in Fig. 3 and Tab. II. In total, eight cases were assessed: $A, B, C, D, A \_$sides, $B \_s i d e s, C \_$sides and A_skirt.

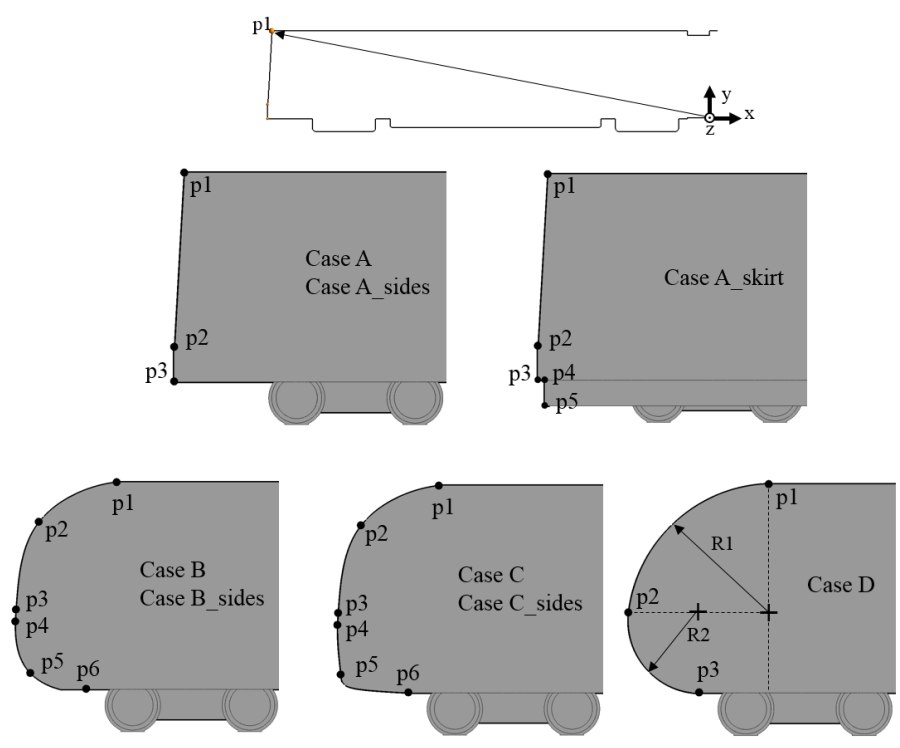

Fig. 2. Schematics of profiles $A, B, C$ and $D$. Point locations and radii are given in Tab. I.

TABLE I

Line type ANd Point LoCATIONS For PRofiles $A, B, C$ And $D$. See FIG. 2 FOR DETAILS.

\begin{tabular}{|c|c|c|c|c|c|c|}
\hline Profile & \multicolumn{2}{|c|}{$\mathbf{A}$} & \multicolumn{2}{|c|}{$\mathbf{B}$} & \multicolumn{2}{|c|}{$\mathrm{C}$} \\
\hline Point & $x / L_{c}$ & $y / H_{t}$ & $x / L_{c}$ & $y / H_{t}$ & $x / L_{c}$ & $y / H_{t}$ \\
\hline p1 & -0.990 & 0.999 & -0.899 & 0.999 & -0.899 & 0.999 \\
\hline p2 & -1.000 & 0.164 & -0.974 & 0.807 & -0.974 & 0.807 \\
\hline p3 & -1.000 & -0.001 & -0.995 & 0.383 & -0.995 & 0.383 \\
\hline p4 & - & - & -1.000 & 0.325 & -1.000 & 0.325 \\
\hline p5 & - & - & -0.982 & 0.076 & -0.993 & 0.085 \\
\hline p6 & - & - & -0.928 & -0.001 & -0.928 & -0.001 \\
\hline Line type & \multicolumn{2}{|c|}{ Straight } & \multicolumn{2}{|c|}{ Spline } & \multicolumn{2}{|c|}{ Spline } \\
\hline Profile & \multicolumn{2}{|c|}{ D } & \multicolumn{2}{|c|}{ Skirt } & & \\
\hline Point & $x / L_{c}$ & $y / H_{t}$ & $x / L_{c}$ & $y / H_{t}$ & & \\
\hline p1 & -0.862 & 0.999 & -0.990 & 0.999 & & \\
\hline p2 & -1.000 & 0.383 & -1.000 & 0.164 & & \\
\hline p3 & -0.928 & -0.001 & -1.000 & -0.001 & & \\
\hline p4 & - & - & -1.000 & -0.001 & & \\
\hline p5 & - & - & -0.993 & -0.126 & & \\
\hline Line type & \multicolumn{2}{|c|}{$\begin{array}{c}\text { Curves } \\
R 1=0.696 H_{t} \\
R 2=0.345 H_{t}\end{array}$} & \multicolumn{2}{|c|}{ Straight } & & \\
\hline
\end{tabular}

\section{NUMERICAL MODELING}

\section{A. Computational Domain and Boundary Conditions}

The computational domain represents a subway train moving through a straight tunnel at a speed of $U_{t}=11.11 \mathrm{~m} . \mathrm{s}^{-1}$. The tunnel, which has a circular arc roof over straight walls, is $4.5 L_{t}$ long with a cross section of height $\sim 1.2 H_{t}$ and width $\sim 1.1 W_{t}$. This results in a blockage ratio of $A_{r}=0.69$. This value remains within the range assessed in different studies on 

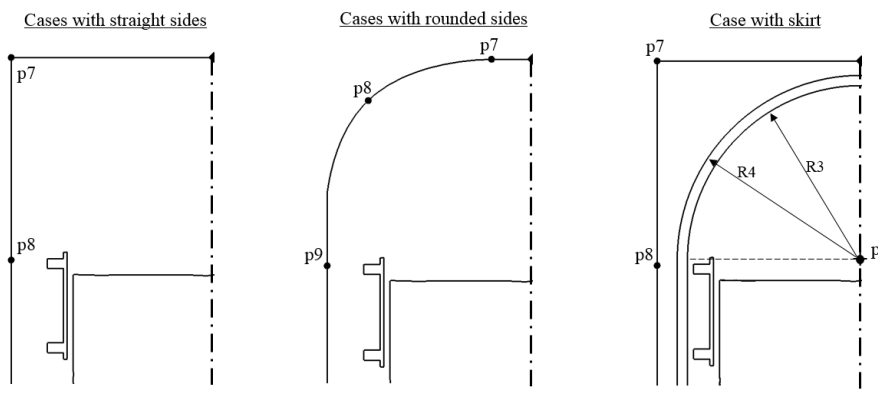

Fig. 3. Schematics of profiles with straight sides, rounded sides and skirt (as seen from underneath). Point locations and radii are given in Tab. II.

TABLE II

LINE TYPE AND POINT LOCATIONS FOR THE PROFILES WITH STRAIGHT SIDES, ROUNDED SIDES AND SKIRT. SEE FIG. 3 FOR DETAILS.

\begin{tabular}{|c|c|c|c|c|c|c|}
\hline Profile & \multicolumn{2}{|c|}{ Straight } & \multicolumn{2}{c|}{ sides } & \multicolumn{2}{c|}{ skirt } \\
\hline Point & $x / L_{c}$ & $z / W_{t}$ & $x / L_{c}$ & $z / W_{t}$ & $x / L_{c}$ & $z / W_{t}$ \\
\hline p7 & -1.000 & 1.000 & -1.000 & 0.200 & -1.000 & 1.000 \\
p8 & -0.905 & 1.000 & -0.981 & 0.800 & -0.905 & 1.000 \\
p9 & - & - & -0.905 & 1.000 & -0.908 & 0.000 \\
\hline \multirow{2}{*}{ Line type } & \multicolumn{2}{|c|}{ Straight } & \multicolumn{2}{|c|}{ Spline } & \multicolumn{2}{c|}{$\begin{array}{c}\text { Curves } \\
R 3=0.8499 W_{t} \\
\end{array}$} \\
\end{tabular}

the influence of the tunnel geometry over the total drag [14]. The blockage ratio is usually smaller for real metro trains between adjacent platforms $(\sim 0.44)$ [5]. The computational domain is striped of small geometrical details (light posts, security bars). Studies on vehicle aerodynamics show that this simplification reduces the resulting total drag by about $5 \%$ [15]. Given the flow configuration, only half of the tunnel was computed in order to reduce computational costs. The domain is shown in Fig. 4 with the train placed at a distance $L_{t}$ of the incoming flow boundary.

Simulations were carried out adopting a frame of reference fixed with the train i.e. the train is static whereas the flow and tunnel move at a relative velocity $U_{t}$ in the opposite direction. Wheel rotation has been neglected in this study as experimental observations report that it has a very weak influence over the total drag (of the order of the experimental uncertainty) [16]. Furthermore, within the context of train aerodynamics, RANS results with and without wheel rotation vary by less than $1 \%$ in terms of the computed drag [17], with an acceptable experimental agreement [18]. This approach has been successfully applied in the aerodynamic analysis of trucks [19]. Hence, as boundary conditions, uniform inlet velocity $U_{t}$ with $0.5 \%$ turbulent intensity was imposed at the incoming flow surface and uniform static pressure (101325 $\mathrm{Pa}$ ) was imposed at the outlet. Model sensibility to the inlet turbulent intensity was assessed by performing preliminary calculations with this parameter increased from $0.5 \%$ up to $5 \%$, resulting in a total drag variation of barely $2 \%$. Experimental studies show that the incoming air onto a train nose has a slightly lower turbulent activity than on its sides, where it is about 10\% [20], while RANS studies in vehicles report a slight increase in pressure drag with higher turbulence activity [21]. The train surfaces were defined as smooth walls while the surrounding tunnel and bottom were defined as smooth walls with a speed $U_{t}$ in the same sense as the flow. Lastly, a symmetry condition was imposed at the vertical mid plane as shown in Fig. 4.

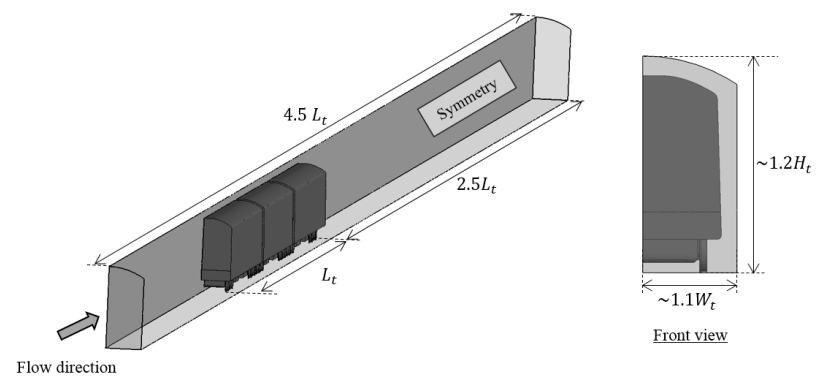

Fig. 4. Schematics of the computational domain.

The computational domain has been discretized using an unstructured mesh conformed by tetrahedral cells with prismatic layers starting from all solid surfaces. Local mesh refinement was imposed in the region around the train and train wake, with a linear grow rate up until the outlet boundary. Six mesh sizes were considered, varying the total cell number between $6.5 \times 10^{6}$ and $18.2 \times 10^{6}$ at an increase rate of $\sim 1.2$. The resulting mesh had $13.1 \times 10^{6}$ elements, with a near train cell length scale of $\sim 0.002 H_{t}$. The prismatic region was conformed by 11 layers, with a first layer thickness of $\sim 0.0008 H_{t}$ and a growing rate of 1.25 , which resulted in average $Y+$ values of, respectively, 40 and 80 for the tunnel and train walls for the baseline case. The definitive meshing parameters were repeated in the generation of all the assessed designs. Details of the final mesh are presented in Fig. 5.

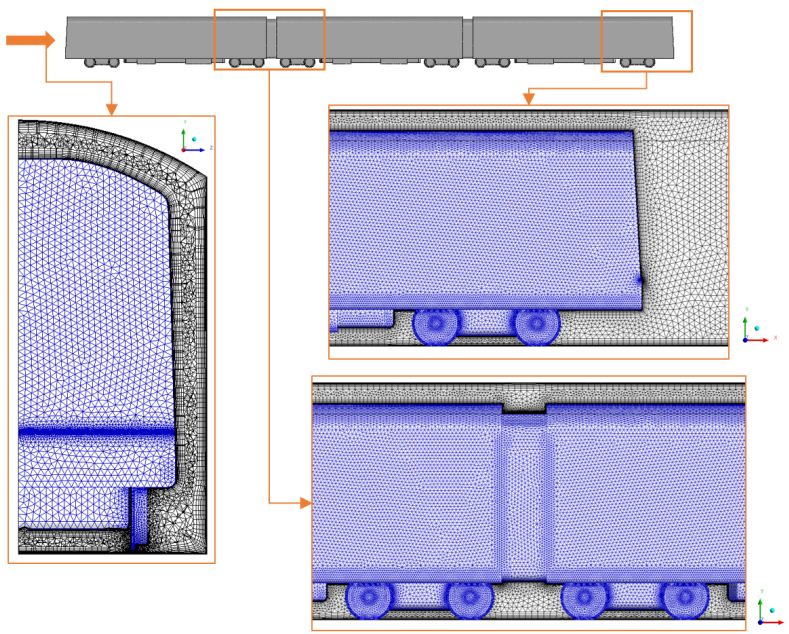

Fig. 5. Details of the resulting mesh for the baseline case.

The Reynolds number based on $H_{t}$ for the flow configuration is $R e_{t}=3.52 \times 10^{6}$. The flow is then highly turbulent. 
The $k-\omega$ SST turbulence model has been chosen in this study, as it is known for its improved treatment of wall bounded flows over the standard $k-\epsilon$ model, offering a good compromise between accuracy, computational costs and numerical stability [22].

\section{B. Numerical Settings}

The RANS equations in steady state were resolved using a finite volume approach. Advective terms were discretized using a second order upwind scheme whereas a least-squares cell based scheme was used for the determination of the spatial gradients. The resulting algebraic system of equations was solved using the SIMPLE algorithm with high order relaxation to soften convergence. The working fluid was air with constant density and viscosity $\left(\rho=\rho_{\text {ref }}\right.$ and $\left.\nu=1.42 \times 10^{-5} \mathrm{~m}^{2} . \mathrm{s}^{-1}\right)$. Convergence was defined as RMS residuals under $10^{-4}$ for all equations plus an invariant skin friction coefficient for more than 1000 iterations. Simulations were performed using the software ANSYS Fluent v18 on the supercomputer Mammouth Parallel 2 from Université de Sherbrooke managed by Compute Canada. For each computation, 6 computing nodes were used, each with $16 G b$ RAM and 2 Intel Xeon E5462 CPUs (4 cores per CPU). A typical steady state computation took about 18 hours from initialization to convergence.

\section{Experimental Validation}

The numerical model has been validated by replicating wind tunnel data of [23], which measured the total drag on different elongated bodies. The case of a circular top edge and squared side edges design was chosen for experimental validation. This body has a total length of $3.94 \mathrm{~m}$ and a frontal facing area of $0.56 \times 0.56 \mathrm{~m}^{2}$. It is subject to a longitudinal wind with nominal velocity of $50 \mathrm{~m} . \mathrm{s}^{-1}$, which results in a Reynolds number of about $1.9 \times 10^{6}$. Thought the experimental configuration has some differences relative to the cases assessed in this study, in particular that the experimental vehicle is not inside a confined tunnel (its blockage rate being 0.021), it permits to evaluate the accuracy of the numerical model for the determination of near-wall flow characteristics and the uncertainty of using a frame of reference fixed with the moving vehicle.

Fig. 6 compares the experimental data [23] and the numerical results using the setup described above. The comparison is made in terms of the dimensionless pressure coefficient $C p$ :

$$
C p=\frac{p-p_{r e f}}{\frac{1}{2} \rho_{r e f} U_{r e f}^{2}}
$$

with the corresponding experimental values $p_{\text {ref }}=p_{\text {exp }}=$ $101325 \mathrm{~Pa}, \rho_{\text {ref }}=\rho_{\text {exp }}=1.225 \mathrm{~kg} \cdot \mathrm{m}^{-3}$ and $U_{\text {ref }}=$ $U_{\text {exp }}=50 \mathrm{~m} . \mathrm{s}^{-1}$. It can be seen that the numerical model has in general a very good agreement with the experimental data, with the most noticeable differences occurring at the leading edge. Numerical accuracy in this region might be affected by flow complexities such as separation and instabilities, as reported in the experimental data source [23].
Moreover, in terms of global results, the total drag coefficient determined with the RANS model $\left(C_{D}\right.$, Eq.(2)) was found to be 0.755 , in excellent agreement with the reported experimental value 0.75 [23]. This validation allows to confidently use the RANS model for the comparison of different geometrical alternatives in terms of aerodynamic performance.
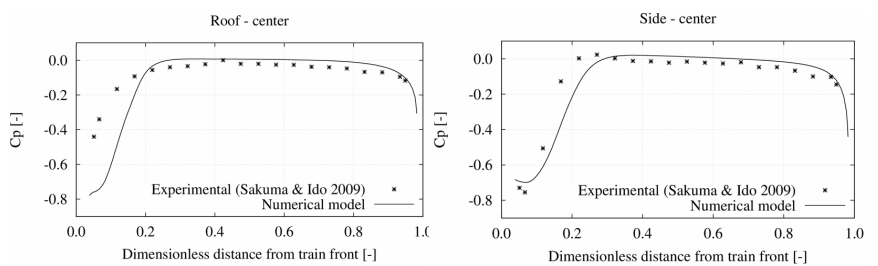

Fig. 6. Comparison in terms of the pressure coefficient with the experimental data of [23].

\section{RESUlts AND Discussion}

The differences between the assessed cases are given in terms of the flow structure around the train and the total aerodynamic drag $D_{\text {total }}$ for each case, which is defined as the contribution of the resulting friction $D_{\text {friction }}$ and pressure $D_{\text {pressure }}$ forces opposing the train movement. These terms were calculated by integrating the wall shear stress and pressure differences on the train surface in the direction of movement. Hence, the total drag coefficient is calculated as:

$$
C_{D}=\frac{D_{\text {total }}}{\frac{1}{2} \rho_{\text {ref }} U_{r e f}^{2} A_{r e f}}
$$

For all train cases $U_{\text {ref }}=U_{t}=11.11 \mathrm{~m} . \mathrm{s}^{-1}$.

\section{A. Friction Drag}

Fig. 7 shows the wall shear stress acting on the train surfaces in the airflow direction. The color legend is such that the higher the value the higher the resistance to movement experimented by the train. As it can be seen in the front face color maps, the shear friction near the train edges is much lower in the baseline case than in the others, a result of rounding the train edges, which delays flow separation and gives a horizontal character to the surface encountering the flow. The difference is particularly important at the trailing edge of geometries $B, C$ and $D$, where the wall shear in the airflow direction is between 3 and 4.5 $\mathrm{Pa}$ for cases with rounded edges whereas for the baseline is almost null. Although these results might seem contrary to the desired effects, it will be shown below that the differences in terms of friction drag across all cases are negligible and, more importantly, their influence on the final drag is very low.

\section{B. Pressure Drag}

Fig. 8 shows contours of the pressure coefficient $C p$ (Eq.(1) with $U_{t}$ instead of $U_{\text {exp }}$ ) at the train front and rear faces. In general, the stagnation point at the train nose varies in size depending on the train design, with the rounded sides cases presenting a smaller front stagnation area. Edge rounding also helps increase the $C p$ at the train rear-face well over 

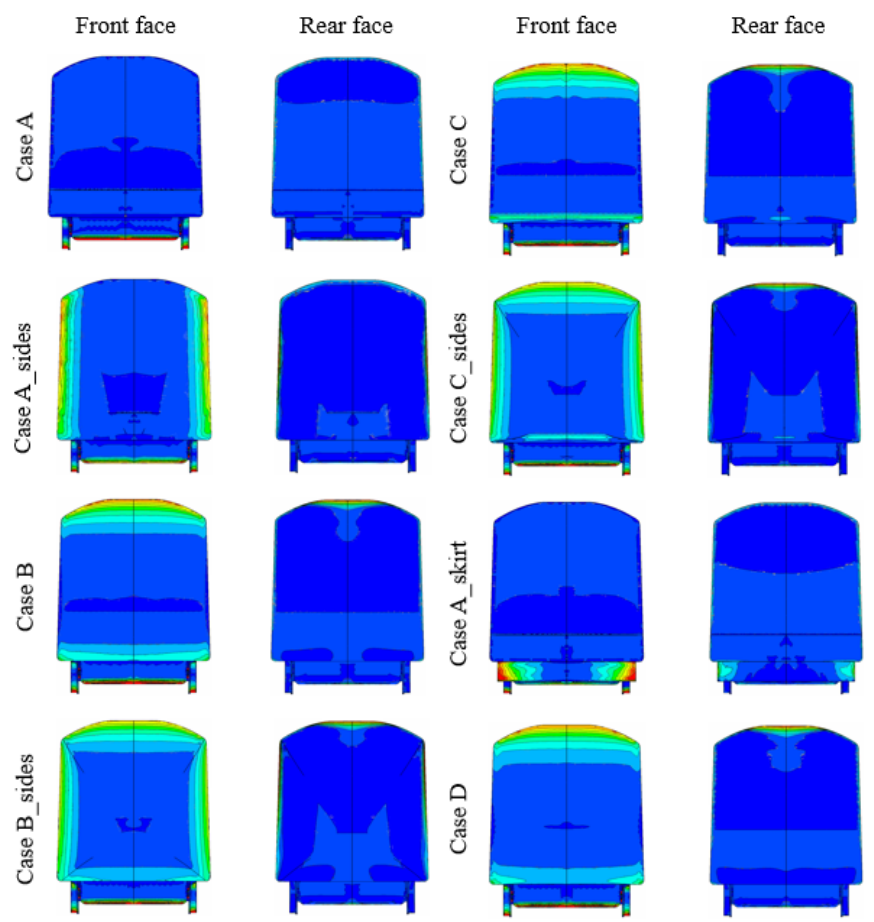

Wall shear stress opposing the train motion $[\mathrm{Pa}]$

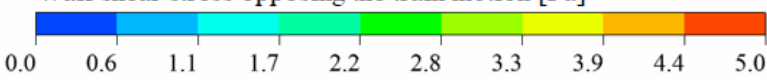

Fig. 7. Wall shear stress on the train surface.

the baseline design value. Reducing the pressure difference between the train front and rear end helps diminish the piston effect as well as the resulting pressure drag. Streamlining the rear face has shown positive effects on the total drag of trailer trucks [24]. Thus, as expected, the baseline design (Case $A$ ) resembling a piston pushing through the tunnel has the highest drag. It must be pointed out that very low $C p$ values, corresponding to very high velocities, were observed at punctual locations, particularly around the train wheels (where the clearance is of a few millimeters) and towards the back of the train. Similar low $C p$ values were obtained when modeling a confined cylinder with the same blockage ratio and Reynolds number (not shown here).

A further effect of rounding off the sides of the train is revealed by comparing streamlines around the body as shown in Fig. 9 for the baseline and $A_{-}$sides cases. In the former, the blunt front face creates a recirculation zone ahead of the first boogie as well as erratic flow trajectories just after the train front, whereas for the latter case the flow between the tunnel wall and the train is much smoother and only fans out at the rear as it redistributes into the tunnel. This improved flow arrangement delays detachment and reduces the pressure difference between the train front and rear faces. This behavior was observed similarly in all rounded side cases.

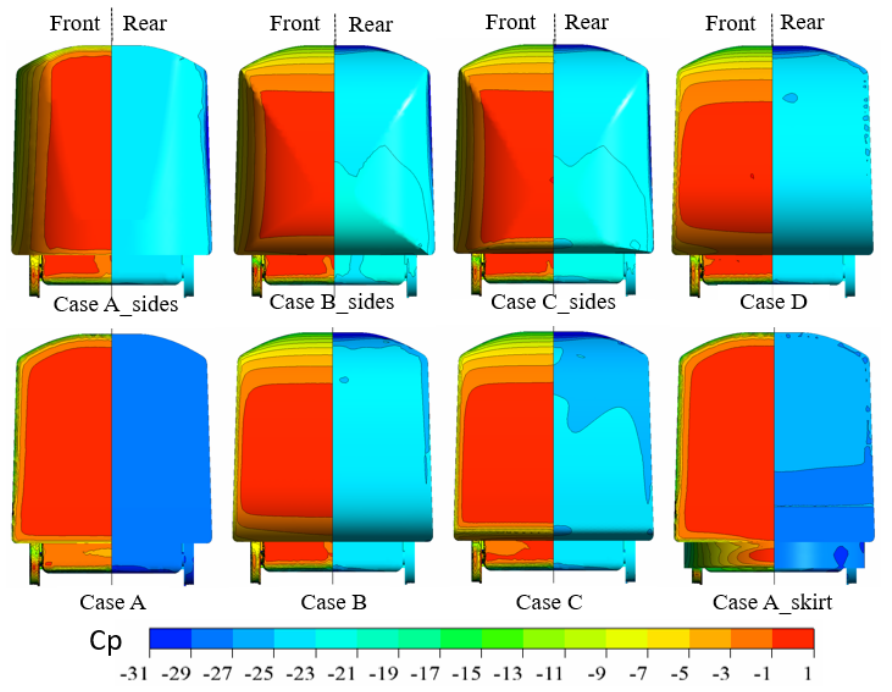

Fig. 8. Contours of local pressure coefficient on the front and rear train faces.
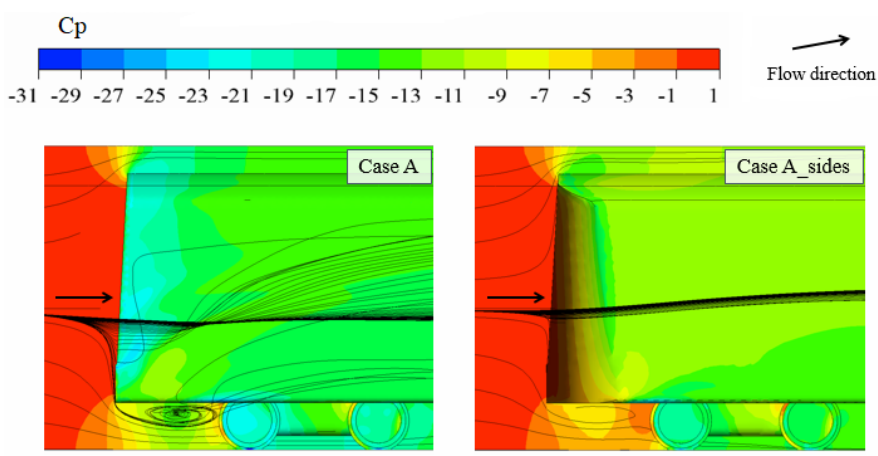

Fig. 9. Streamlines following the air velocity around the train. Comparison between cases $A$ (baseline) and A_sides.

\section{Total Drag Comparison}

As expected, the baseline case is the worst design in terms of aerodynamics forces $\left(C_{D_{A}}=9.53\right)$, followed by the design A_skirt which is just $5 \%$ better, albeit the modification in this case is very simple. Next are cases $C, B$ and $D$ which have, in average, a $15 \%$ lower drag coefficient. For all the assessed cases, the rounded side versions are consistently better than their relative originals. This is particularly noticeable in case A_sides, which reduces the baseline drag by $15 \%$, even lower than that achieved by the drastic top and bottom rounding off of case $D$. The best performance is obtained in case $C_{-}$sides, with $C_{D_{C_{\text {sides }}}}=7.61$.

A decomposition of the contribution of $D_{\text {friction }}$ and $D_{\text {pressure }}$ to the total drag exerted on the train is shown in Fig. 10. It is observed that the friction drag augmentation with the design modifications mentioned previously is negligible as $D_{\text {friction }}$ represents only about $5 \%$ of $D_{\text {total }}$ and the variation amongst all cases is of less than $3 \%$.

Concerning the pressure drag, it was verified during the analysis that the pressure field behind the train is very similar among all cases. Thus, it is clear that an important benefit is 
obtained by reducing the size of the maximum $C p$ area at the front of the train, which generates a lower pressure gradient between the front and rear ends. This is clearly reflected in Fig. 10, where the total drag varies directly with the proportion of $D_{\text {pressure }}$ for each case.

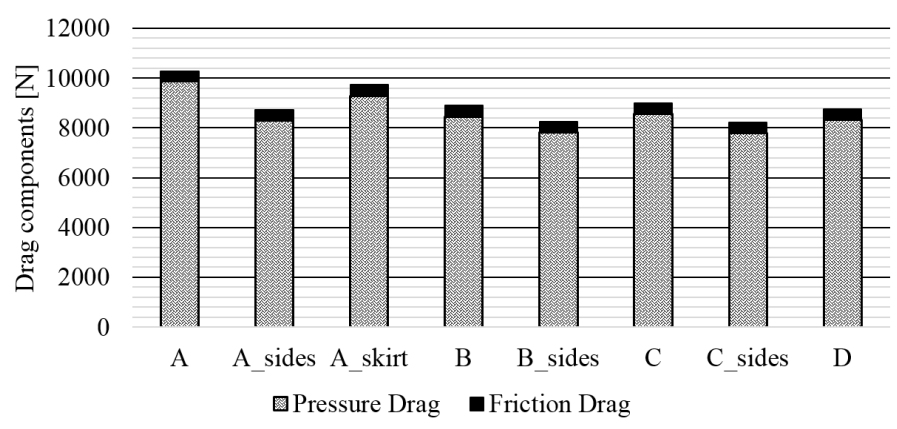

Fig. 10. Contribution of friction and pressure to total drag for all cases.

\section{CONCLUSIONS}

The aerodynamic performance of a typical metro train geometry has been compared with 7 design alternatives using an incompressible RANS solver using the $k-\omega$ SST turbulence model. All cases were assessed considering a train moving at constant speed through a tunnel, which allows to use a frame of reference moving with the train. Experimental validation versus the data of [23] shows that the model accurately captures the flow field in the near-train region.

Case $C_{-}$sides offers the greatest drag reduction over the baseline, 21\%. Model A_skirt, being essentially the same geometry as case $A$ with a deflector around the bogies, offers the lowest drag reduction (5\%). Decomposition of the total drag shows that the most relevant component is $D_{\text {pressure }}$, which is about $95 \%$ of the total aerodynamic resistance for all cases. Thus, the best performing alternatives are those managing to minimize the stagnation area at the front of the train, namely cases $A \_$sides, $B \_$sides and $C \_$sides. Relative to the baseline, cases $B, C$ and $D$ offer an average drag reduction of $14 \%$, whereas for cases $A \_$sides, $B \_$sides and $C \_$sides the average improvement is $19 \%$. This represents an estimated gain of $2 \%$ in terms of total energy savings for the whole system, based on subway energy accounting metrics [4]. Rounding the front face edges increases the shear friction on the train surface. However the average difference in wall shear stress between the modified cases and the baseline is less than $3 \%$. Furthermore the friction coefficient represents only about $5 \%$ of the total train drag, making the increased surface of streamlined designs a minor concern. All cases with rounded sides showed a smoother flow arrangement around the train, as well as a lower front-to-back pressure difference, suggesting the usefulness of this modification to mitigate the train-induced piston effect in the tunnel.

\section{REFERENCES}

[1] Z. Guo, and N. H. Wilson, "Assessing the cost of transfer inconvenience in public transport systems: A case study of the London underground," Transport. Res. A Policy Pract., vol. 45 (2), pp. 91-104, 2011.
[2] J. Kim, and K. Kim, "Experimental and numerical analyses of traininduced unsteady tunnel flow in subway," Tunn. Undergr. Sp. Tech., vol. 22 (2), pp. 166-172, 2007.

[3] W. Woods, and C. Pope, "A generalised flow prediction method for the unsteady flow generated by a train in a single-track tunnel," J. Wind Eng. Ind. Aerod., vol. 7 (3), pp. 331-360, 1981.

[4] A. González-Gil, R. Palacin, P. Batty, and J. Powell, "A systems approach to reduce urban rail energy consumption," Energ. Convers. Manage., vol. 80, pp. 509-524, 2014.

[5] J. Niu, D. Zhou, X. Liang, T. Liu, and S. Liu, "Numerical study on the aerodynamic pressure of a metro train running between two adjacent platforms," Tunn. Undergr. Sp. Tech., vol. 65, pp. 187-199, 2017.

[6] S. Jakirlic, L. Kutej, B. Basara, and C. Tropea, "Computational study of the aerodynamics of a realistic car model by means of RANS and hybrid RANS/LES approaches," SAE Int. J. Passeng. Cars Mech. Syst., vol. 7, pp. 559-574, 2014

[7] J. Zhu, and Z. Hu, "Flow between the train underbody and trackbed around the bogie area and its impact on ballast flight," J. Wind Eng. Ind. Aerod., vol. 166, pp. 20-28, 2017.

[8] F. E. Camelli, G. Byrne, and R. Löhner, "Modeling subway air flow using CFD,” Tunn. Undergr. Sp. Tech., vol. 43, pp. 20-31, 2014.

[9] J.-K. Choi, and K.-H. Kim, "Effects of nose shape and tunnel crosssectional area on aerodynamic drag of train traveling in tunnels," Tunn. Undergr. Sp. Tech., vol. 41, pp. 62-73, 2014.

[10] C. Baker, "The flow around high speed trains," J. Wind Eng. Ind. Aerod., vol. 98 (6-7), pp. 277-298, 2010.

[11] W. Yang, E. Deng, M. Lei, P. Zhang, and R. Yin, "Flow structure and aerodynamic behavior evolution during train entering tunnel with entrance in crosswind," J. Wind Eng. Ind. Aerod., vol. 175, pp. 229-243, 2018.

[12] Y. Shuanbao, G. Dilong, S. Zhenxu, Y. Guowei, and C. Dawei, "Optimization design for aerodynamic elements of high speed trains," Comput. Fluids, vol. 95, pp. 56-73, 2014.

[13] H. Martini, B. Bergqvist, L. Hjelm, and L. Lofdahl, "Aerodynamic investigation of gap treatment-and chassis skirts strategies for a novel long-haul vehicle combination," SAE Int. J. Commer. Veh., vol. 5, pp. 616-627, 2012.

[14] D. Cross, B. Hughes, D. Ingham, and L. Ma, "A validated numerical investigation of the effects of high blockage ratio and train and tunnel length upon underground railway aerodynamics," J. Wind Eng. Ind. Aerod., vol. 146, pp. 195-206, 2015.

[15] A. I. Heft, T. Indinger, and N. A. Adams, "Experimental and numerical investigation of the DrivAer model," in: ASME 2012 Fluids Engineering Division Summer Meeting, Rio Grande, Puerto Rico, USA, Paper FEDSM2012-72272, pp. 41-51, 2012.

[16] R. Crane, and C. Morton, "Drag and side force analysis on bicycle wheel-tire combinations," J. Fluid. Eng., vol. 140 (6), 061205, 2018.

[17] J. Zhang, J.-J. Li, H.-Q. Tian, G.-J. Gao, and J. Sheridan, "Impact of ground and wheel boundary conditions on numerical simulation of the high-speed train aerodynamic performance," J. Fluid. Struct., vol. 61, pp. 249-261, 2016.

[18] A. Khayrullina, B. Blocken, W. Janssen, and J. Straathof, "CFD simulation of train aerodynamics: train-induced wind conditions at an underground railroad passenger platform,” J. Wind Eng. Ind. Aerod., vol. 139, pp. 100-110, 2015.

[19] M. Lateb, H. Fellouah, "Numerical analysis of lateral skirts performance on drag force of a semi-trailer truck," in: ASME 2018 5th Joint USEuropean Fluids Engineering Conference, Montreal, Quebec, Canada, Paper FEDSM2018-83457, 2018.

[20] C. Baker, "A review of train aerodynamics part 1-fundamentals," Aeronaut. J., vol. 118 (1201), pp. 201-228, 2014.

[21] C. Fu, M. Uddin, and C. Selent, "The effect of inlet turbulence specifications on the RANS CFD predictions of a Nascar GEN-6 racecar," Tech. rep., SAE Technical Paper, 2018.

[22] A. Mattana, S. Salvadori, T. Morbiato, and C. Borri, "On the groundvehicle induced flows and obstacle interaction for energy harvesting purposes," J. Wind Eng. Ind. Aerod., vol. 124, pp. 121-131, 2014.

[23] Y. Sakuma, and A. Ido, "Wind tunnel experiments on reducing separated flow region around front ends of vehicles on meter-gauge railway lines," Q. Rep. RTRI, vol. 50 (1), pp. 20-25, 2009.

[24] L. Salati, F. Cheli, and P. Schito, "Heavy truck drag reduction obtained from devices installed on the trailer," SAE Int. J. Commer. Veh., vol. 8, pp. 747-760, 2015. 